\title{
Functional Characterization of fst 1 in Fusarium verticillioides During Colonization of Maize Kernels
}

\author{
Hun Kim and Charles P. Woloshuk \\ Department of Botany and Plant Pathology, Purdue University, West Lafayette, IN 47907, U.S.A. \\ Submitted 23 March 2010. Accepted 10 September 2010.
}

\begin{abstract}
The putative hexose transporter gene $f$ st 1 in Fusarium verticillioides was identified previously by microarray analysis as a gene that was more highly expressed during colonization of autoclaved maize endosperm than germ. In contrast to a previous study, in which disruption of $f$ st 1 did not affect growth of the pathogen on autoclaved maize kernels, in the current study, we demonstrated that disruption of $f_{s t} 1$ delayed growth and symptom development on wounded maize ears. Characterization of the $f$ st 1 promoter revealed that regulation of $f s t 1$ expression was similar to that of fumonisin biosynthetic (fum) genes; expression was highest during growth on endosperm tissue and repressed by elevated concentrations of ammonium in the growth medium. With a fluorescent tag attached to FST1, the protein localized transiently to the periphery of the cells near the plasma membrane and in vacuole-like structures, suggesting that membrane-localized FST1 was internalized and degraded in vacuoles. Expression of $\boldsymbol{f}$ st 1 in a yeast strain lacking hexose transporter genes did not complement the yeast mutation, suggesting that FST1 does not transport glucose, fructose, or mannose. The results indicate a functional role for FST1 in pathogenesis during the colonization of living kernels.
\end{abstract}

Fusarium verticillioides (Sacc.) Nirenberg (teleomorph: Gibberella moniliformis) is a plant pathogen that causes ear, seedling, and stalk diseases in maize. In infected maize kernels, this fungus produces fumonisin mycotoxins that can adversely affect human and animal health. Of the 28 fumonisin analogs that have been isolated and characterized, fumonisin $\mathrm{B}_{1}\left(\mathrm{FB}_{1}\right)$ is most abundant in naturally contaminated maize (Rheeder et al. 2002). $\mathrm{FB}_{1}$ specifically disrupts sphingolipid metabolism by inhibiting ceramide synthase, and its action is associated with diseases in mammals such as pulmonary edema, neural tube defects, and cancer (Desai et al. 2002). Because of the health risks associated with its consumption, $\mathrm{FB}_{1}$ contamination of maize is a food safety issue worldwide.

The study described here focuses on one of the major nutrient factors within the maize kernel that affects $\mathrm{FB}_{1}$ biosynthesis in F. verticillioides; namely, carbon source. $\mathrm{FB}_{1}$ production is highest in endosperm tissue and in a medium containing amylopectin as the sole carbon source (Bluhm and Woloshuk 2005; Shim et al. 2003), and $\alpha$-amylase appears to be required for starch degradation during growth on the endosperm (Bluhm and Woloshuk 2005). Acquisition of the starch-derived sugars by the fungus requires sugar transporters. The genome

Corresponding author: C. P. Woloshuk; Telephone: 765494 3450; Fax: 765494 0363; E-mail: woloshuk@purdue.edu of $F$. verticillioides has at least 147 putative genes that encode proteins with significant similarity to sugar transporters, none of which has been biochemically characterized (Bluhm et al. 2008). The $F$. verticillioides gene $f s t 1$ was identified by comparative microarray analysis of RNA isolated from colonized maize germ and endosperm. The gene is located on chromosome 10 of the F. verticillioides genome (FVEG_08441.3, supercontig 11: 403345 to 405266 ), has one intron (46 bp), and encodes a 574-amino-acid protein with 12 putative transmembrane domains. Of the 50 microarray probes specific for putative sugar transporter genes, $f s t 1$ was one of six genes that are more highly expressed during growth of $F$. verticillioides in maize endosperm tissue compared with germ tissue (Bluhm et al. 2008). Expression of $f_{s t} 1$ and several other putative sugar transporters was reduced in a strain with a mutation in $z f r l$, a gene encoding a putative zinc-finger transcription factor. Disruption mutants of $f s t 1$ have no discernible effect on growth or development when cultured on defined media and on autoclaved maize kernels; however, $\mathrm{FB}_{1}$ production is severely reduced (Bluhm et al. 2008).

Bluhm and associates (2008) hypothesized that FST1 functions as an environmental sensor that is required for high levels of $\mathrm{FB}_{1}$ production. The mechanism for sugar sensing has been studied extensively in yeast (Santangelo 2006). Snf3p and Rgt $2 p$ are proteins located in the yeast plasma membrane which sense low and high external glucose concentrations, respectively (Ozcan et al. 1996, 1998). The amino acid sequences of these proteins have high identity to sugar transporters; however, they have no detectable transport activity. Sensing sugars in filamentous fungi is still poorly understood. The Neurospora crassa gene rco3 is one of the only described sugar sensors in this group of fungi (Madi et al. 1997). Deletion of rco3 affects the expression of glucose-repressible genes and conidial development (Madi et al. 1997). The molecular mechanism by which RCO3 functions as a sensor remains to be determined.

In the study presented here, we investigated the function of $f s t 1$ in $F$. verticillioides, addressing questions about its effect on pathogenicity, the conditions that activate transcription, possible hexose transporter activity, and cellular localization. The results indicate that FST1 is localized to the fungal cell membrane and that the protein has a role in pathogenicity.

\section{RESULTS}

Gene $f$ st 1 is required for wild-type levels of kernel colonization and $\mathrm{FB}_{1}$ production.

Maize ears were wound inoculated with the wild type, $\Delta f s t 1$, and complemented strain $\Delta f s t$-comp2 (formerly FST-COMP2) 
(Bluhm et al. 2008). After 7 days, kernels inoculated with the wild type and $\Delta f s t 1$-comp2 exhibited discoloration and extensive mycelial growth on the surface of the kernels (Fig. 1). On ears inoculated with $\Delta f s t 1$, kernels were less discolored and visible mycelial growth was restricted to the wound sites (Fig. 1). The kernels inoculated with the wild type and $\Delta f s t 1$-comp 2 also had chalky endosperms and discolored germs. In contrast, the endosperm of kernels inoculated with $\Delta f s t 1$ remained firm and yellow, and germ tissues appeared unaffected, although the tissue surrounding the wound site was discolored. Microscopic examination confirmed that mycelia and conidia were present throughout the endosperm of kernels infected by the wild type and $\Delta f s t 1$-comp2. However, mycelia of $\Delta f s t 1$ were visible only at the wound site. Consistent with these observations, kernels inoculated with $\Delta f s t 1$ contained $50 \%$ less ergosterol after 7 days than kernels inoculated with the wild type or

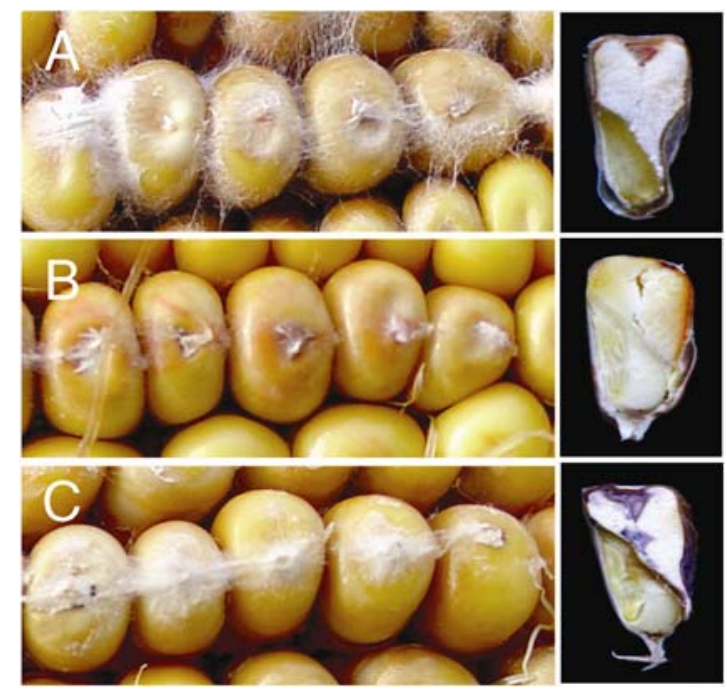

Fig. 1. Effect of $f_{s t} 1$ on maize kernel colonization and symptoms induced by Fusarium verticillioides inoculation. Conidia of $\mathbf{A}$, the wild type; $\mathbf{B}$, $\Delta f s t 1$; and $\mathbf{C}, \Delta f s t 1$-comp2 were inoculated into maize ears by a needle. Pictures were taken at 7 days after inoculation. Right panel shows a cross section through a representative kernel.

Table 1. Effect of FST1 on fumonisin $\mathrm{B}_{1}\left(\mathrm{FB}_{1}\right)$ production and growth during colonization of maize ears ${ }^{\mathrm{a}}$

\begin{tabular}{lccccc}
\hline & \multicolumn{2}{c}{$\mathbf{7}$ days } & & \multicolumn{2}{c}{$\mathbf{1 4}$ days } \\
\cline { 2 - 3 } \cline { 5 - 6 } Fungal strain & $\mathbf{F B}_{\mathbf{1}}^{\mathbf{b}}$ & Growth $^{\mathbf{c}}$ & & $\mathbf{F B}_{\mathbf{1}}^{\mathbf{b}}$ & Growth $^{\mathbf{c}}$ \\
\hline Wild type & $44 \pm 21$ & $147 \pm 42$ & & $91 \pm 27$ & $331 \pm 56$ \\
$\Delta f s t 1$ & $\mathrm{nd}$ & $71 \pm 17$ & & nd & $275 \pm 65$ \\
$\Delta f s t 1$-comp2 & $39 \pm 2$ & $143 \pm 48$ & & $48 \pm 4$ & $334 \pm 78$ \\
\hline
\end{tabular}

${ }^{\text {a }}$ Wounded kernels inoculated by the wild type, fstl disruption mutant $(\Delta f s t 1)$, and complementation strain $\left(\Delta f s t 1\right.$-comp2) were collected for $\mathrm{FB}_{1}$ and ergosterol analysis; $\mathrm{nd}=$ not detected. Limits of detection for $\mathrm{FB}_{1}$ were $4 \mu \mathrm{g} / \mathrm{g}$ of maize kernels and for ergosterol were $10 \mu \mathrm{g} / \mathrm{g}$ of maize kernels.

${ }^{\mathrm{b}} \mathrm{FB}_{1}$ measured in micrograms per gram of maize kernels \pm standard deviation.

${ }^{c}$ Ergosterol measured in micrograms per gram of maize kernels \pm standard deviation.
$\Delta f$ stl-comp2 (Table 1 ). In addition, $\mathrm{FB}_{1}$ accumulated in kernels inoculated with the wild type or $\Delta f s t 1$-comp2 but not in kernels inoculated with $\Delta f s t l$ (Table 1). With longer incubation (14 days), disease symptoms of kernels inoculated with $\Delta f s t 1$ were similar to those on kernels inoculated with the wild type at 7 days, with extensive mycelial growth on the surface of the kernels, chalky endosperm tissues, and discolored germ tissues. In contrast, kernels inoculated with the wild type or $\Delta f s t$ 1-comp2 were completely rotted at 14 days after inoculation. Furthermore, ergosterol content from kernels infected with $\Delta f s t l$ was $20 \%$ less than kernels infected with the wild type, indicating that FST1 affects the rate of disease development (Table 1). However, $\mathrm{FB}_{1}$ was not detectable in the $\Delta f s t 1$ infected kernels at 14 days after inoculation.

\section{Conditions that activate $f$ st 1 transcription.}

Bluhm and associates (2008) demonstrated that transcripts of $f_{s t} l$ were higher in colonized endosperm tissue compared with germ tissue. To determine whether this regulation resides in the promoter of $f s t 1$, strain FPC-896 was generated by transforming the wild-type strain with a construct containing the $f_{s t} 1$ promoter region (896 bp) fused to the $m$ Cherry-FP reporter (Fig. 2). At 4 days after inoculation of autoclaved endosperm and germ tissues with strain FPC-896, fluorescence was observed in mycelia growing on endosperm tissue but not on germ (Fig. 3). For more detailed functional analysis of the $f_{s t} l$ promoter, smaller segments $(509,247$, and $96 \mathrm{bp})$ were fused to the $m$ Cherry-FP gene to generate strains FPC-509, FPC247, and FPC-96 (Fig. 2). Fluorescence was observed in mycelia of strain FPC-509 after 4 days of growth on autoclaved maize kernels but not in mycelia of strains FPC-247 and FPC96. Also, in defined liquid medium with glucose (DLG), mycelia of FPC-509 exhibited fluorescence but mycelia of FPC-247 and FPC-96 did not (data not shown). These results indicate that the region between -248 and -509 bp is essential for tissue-specific promoter activity.

The regulation of $\mathrm{FB}_{1}$ biosynthesis is greatly influenced by nitrogen source. Previous research demonstrated that excessive amounts of ammonium repress $\mathrm{FB}_{1}$ production (Kim and Woloshuk 2008; Shim and Woloshuk 1999). To determine the effect of nitrogen on expression of $f s t 1$, mycelium of FPC-896 was transferred to a medium with and without ammonium phos-
Germ

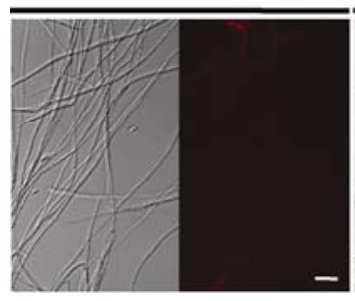

\section{Endosperm}

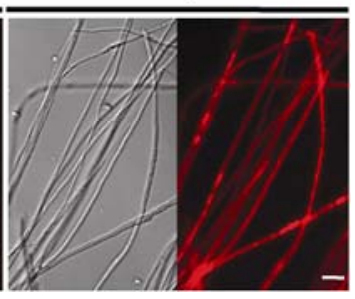

Fig. 3. Expression of $f s t 1$ promoter:: $m$ Cherry-FP during colonization of maize kernel tissues. Strain FPC-896 was grown on germ and endosperm tissues for 4 days. In each panel, left pictures are a DIC image and right pictures are a fluorescent image. Size bars indicate $10 \mu \mathrm{m}$.

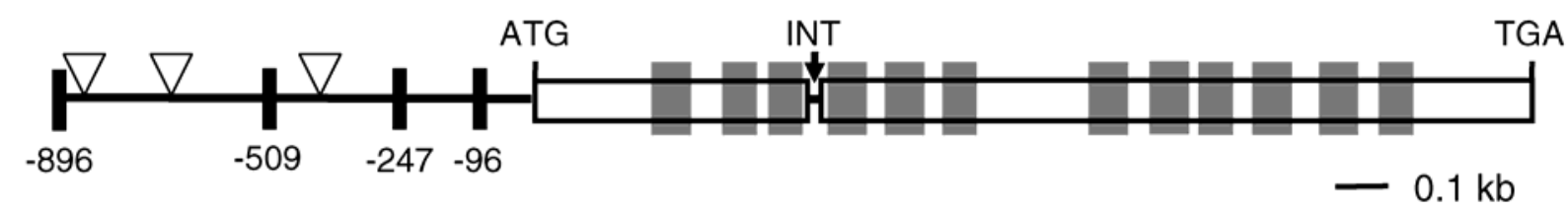

Fig. 2. Structure of $f_{s t 1}$. Gray boxes indicate the coding regions for the putative transmembrane domains. Black bars indicate the positions of forward primers for the amplification of $5^{\prime}$ untranslated regions of $896,509,247$, or $96 \mathrm{bp}$ used in promoter analysis. White triangles indicate putative AREA binding sites $(-393,-737$, and $-862 \mathrm{bp})$. The intron was designated as INT. 
phate. After 3 days of incubation, fluorescent mycelia were observed, and $\mathrm{FB}_{1}$ was measured in the cultures both with and without ammonium phosphate (Table 2). As controls, two additional strains were examined: NIAD-GUS, which contains the promoter of the $F$. verticillioides nitrate reductase gene (niaD) fused to $\beta$-glucuronidase (GUS), and FUM8-GFP, which contains the fum 8 promoter fused to green fluorescent protein (GFP). Similar to FPC-896, expression of reporters and $\mathrm{FB}_{1}$ production was suppressed by ammonium (Table 2). These results indicate that $f s t 1$ expression is subject to nitrogen metabolite repression similar to the fum genes and nitrate reductase gene (Kim and Woloshuk 2008).

\section{Hexose transporter activity.}

FST1 contains two G-R-R(K) motifs, a typical sugar transporter signature, located at amino acid positions 141 to 143 (between the second and third transmembrane domains) and 387 to 389 (between the eighth and ninth transmembrane domains). A BLAST search with the FST1 sequence indicated similarity to putative sugar transporters from other fungi, including Fusarium spp., Magnaporthe grisea, and Aspergillus nidulans. The highest similarities were to a hypothetical fructose transporter in F. graminearum (FGSG_03168.3, 90\%), a

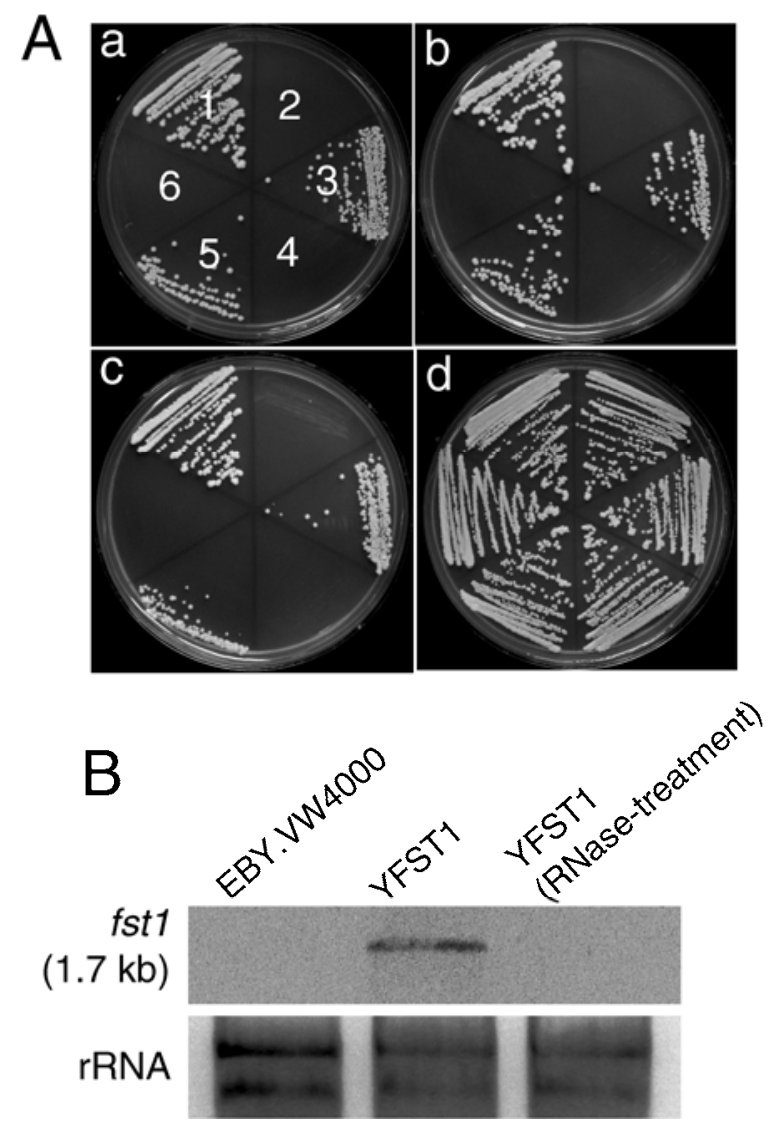

Fig. 4. Expression of $f_{s t} 1$ in Saccharomyces cerevisiae EBY.VW4000. A, Growth of yeast strains was tested on yeast-extract peptone medium containing glucose (a), fructose (b), mannose (c), or maltose (d). Pictures were taken at 3 days after inoculation: 1, wild-type $S$. cerevisiae YRG-2; 2 , strain EBY.VW4000; 3, strain EBY.VW4000 transformed with yeast $H X T 1$; 4, strain EBY.VW4000 transformed with $f s t 1$; 5, strain EBY.VW4000 transformed with $f_{s t 13}$; and 6, strain EBY.VW4000 transformed with p427TEF (empty vector). B, Polymerase chain reaction (PCR) analysis of $f s t 1$ full-length transcript from yeast strains. The agarose gel shows $1.7-\mathrm{kb}$ PCR products amplified from cDNA of YFST1 but no product from cDNA of EBY.VW 4000 and YFST1 (RNase-treatment). As an RNA loading control, the ribosomal RNA (rRNA) on the gel was photographed under UV light. hypothetical fructose symporter in $F$. oxysporum (FOXG $10063.2,98 \%$ ), a hypothetical arabinose-proton symporter in M. grisea (MGG_01373.6, 73\%), and a hypothetical glucose transporter in A. nidulans (ANID_02794.1, 80\%).

To address the question of FST1 function, a $f$ st 1 cDNA was expressed in a strain of Saccharomyces cerevisiae lacking 17 hexose transporters (Wieczorke et al. 1999). A strain expressing $f s t 1$ (YFST1) failed to grow on media containing glucose, fructose, or mannose (Fig. 4A). Analysis of the RNA in YFST1

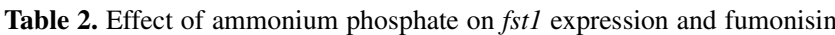
$\mathrm{B}_{1}\left(\mathrm{FB}_{1}\right)$ production $^{\mathrm{a}}$

\begin{tabular}{lcccccc}
\hline & \multicolumn{2}{c}{ DLG } & & \multicolumn{2}{c}{ DLG + AP } \\
\cline { 2 - 3 } \cline { 5 - 6 } Fungal strain $^{\mathbf{b}}$ & Reporter $^{\mathbf{c}}$ & $\mathbf{F B}_{\mathbf{1}}{ }^{\mathbf{d}}$ & & Reporter $^{\mathbf{c}}$ & FB $_{\mathbf{1}}{ }^{\mathbf{d}}$ \\
\hline FPC-896 & + & $116 \pm 18$ & & - & nd \\
NIAD-GUS & + & $103 \pm 36$ & & - & nd \\
FUM8-GFP & + & $117 \pm 23$ & & - & nd \\
\hline
\end{tabular}

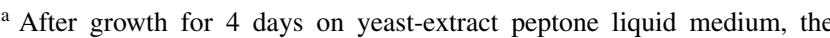
mycelia were transferred to either defined liquid medium (DLG) or DLG plus ammonium phosphate (AP). Gene expression and $\mathrm{FB}_{1}$ were determined 3 days later with three biological replications; nd $=$ not detected. Limit of detection for $\mathrm{FB}_{1}$ was $5 \mu \mathrm{g} / 10 \mathrm{ml}$ of medium.

${ }^{\mathrm{b}} \mathrm{NIAD}=$ nitrate reductase, $\mathrm{GUS}=\beta$-glucuronidase, and $\mathrm{GFP}=$ green fluorescent protein.

${ }^{c}$ Reporter response: $+=$ fluorescence or blue GUS staining visible and $-=$ no fluorescence or GUS staining visible.

${ }^{\mathrm{d}} \mathrm{FB}_{1}$ measured in micrograms per $10 \mathrm{ml}$ of medium \pm standard deviation.

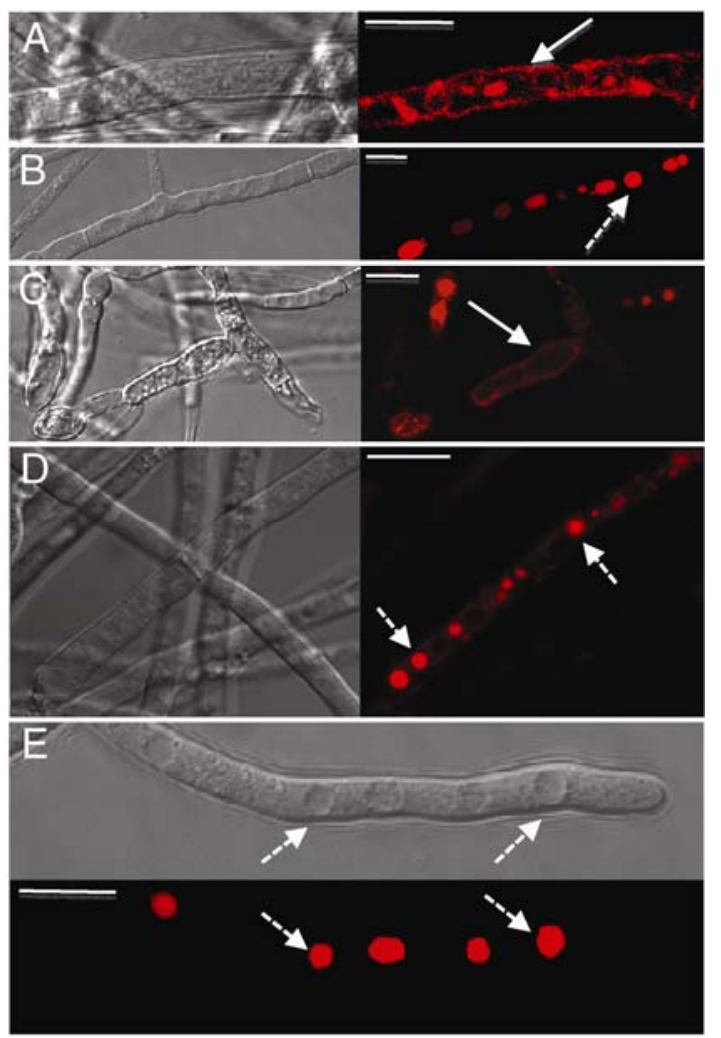

Fig. 5. Cellular localization of FST1 in Fusarium verticillioides. Strain $\Delta f s t 1$-FCT11 was grown on yeast-extract peptone medium before transferring the mycelia to $\mathbf{A}, \mathbf{B}$, and $\mathbf{E}$, amylopectin medium and $\mathbf{C}$ and $\mathbf{D}$, glucose medium. Mycelia were examined with a confocal laser scanning microscope and photographed at $\mathbf{A}, \mathbf{C}$, and $\mathbf{D}, 48 \mathrm{~h}$ and $\mathbf{B}$ and $\mathbf{E}, 72 \mathrm{~h}$ after transferring mycelia. Fluorescent microscope images are shown in the right panels (A to D) and lower panel (E). Light microscope images are in the left panels (A to D) and upper panel (E). Solid arrows indicate fluorescence visible at the periphery of the cells near the plasma membrane. Dashed arrows indicate vacuole-like vesicles. All size bars indicate $10 \mu \mathrm{m}$. 
indicated that a full-length transcript was produced (Fig. 4B). In contrast, strain YHXT1 expressing the yeast hexose transporter gene $(H X T 1)$ was able to grow on these media (Fig. $4 \mathrm{~A})$. The putative hexose transporter gene $(f s t 13)$ from $F$. verticillioides was identified in expressed sequence tag libraries (Brown et al. 2005). Expression of $f_{s t 13}$ was able to complement the yeast mutation, indicating that $f s t 13$ encodes a hexose transporter (Fig. 4A). These results further suggest that FST1 does not function as a hexose transporter.

\section{Cellular localization of FST1.}

The cellular localization of FST1 was investigated by fusion of FST1 to the fluorescent protein mCherry-FP to create the fusion construct FCT11, which was transformed into strain $\Delta f s t 1$. The strain expressing the fusion protein $(\Delta f s t 1$-FCT11) produced fumonisin on autoclaved maize kernels $\left(\mathrm{FB}_{1}\right.$ at $75 \pm$ $10 \mu \mathrm{g} / \mathrm{g}$ of maize kernels), approximately half the amount produced by the wild type ( $\mathrm{FB}_{1}$ at $144 \pm 6 \mu \mathrm{g} / \mathrm{g}$ of maize kernels). Under the same conditions, $\Delta f s t 1$ produced no detectable $\mathrm{FB}_{1}$. These results indicate that the FST1 fusion construct was functional. Based on ergosterol content, the construct did not impact the growth on autoclaved maize kernels; the mean $( \pm$ standard deviation) levels of ergosterol in wild-type, $\Delta f$ st 1 , and $\Delta f$ stl-FCT11 cultures by three replications were $400 \pm 34,378$ \pm 89 , and $384 \pm 26 \mu \mathrm{g} / \mathrm{g}$ of maize kernel culture, respectively. Strain $\Delta f s t 1$-FCT11 was examined with a confocal fluorescent microscope during growth on autoclaved kernels, yeast extract-peptone (YEP), glucose, and amylopectin media. On all but the YEP medium, fluorescence was visible at the periphery of the cells near the plasma membrane and inside vacuole-like vesicles (Fig. 5). Only a subset of the hyphae expressed fluorescence, and the expression appeared along the full length of these hyphae, including new branch points. When transferred from YEP to glucose medium, fluorescence was first observed after $24 \mathrm{~h}$. Fluorescence remained visible up to $48 \mathrm{~h}$ but was not detectable at $72 \mathrm{~h}$. When the mycelia were transferred from YEP to amylopectin medium, fluorescence was visible at 48, 72, and $96 \mathrm{~h}$. These results were consistent with quantitative reverse-transcription polymerase chain reaction (PCR) data indicating that expression of $f s t l$ was highest at $24 \mathrm{~h}$ in the glucose medium and $48 \mathrm{~h}$ in the amylopectin medium (data not shown). Analysis of the sugar content in the two media indicated that $75 \%$ of the sugar in the glucose medium was consumed within $24 \mathrm{~h}$ after resuspension and all sugar was exhausted by $48 \mathrm{~h}$. In contrast, consumption of sugar was more gradual in the amylopectin medium, with $25 \%$ of the original sugar content still remaining at $96 \mathrm{~h}$.

\section{DISCUSSION}

In the previous study by Bluhm and associates (2008), no growth defects were measured in the $f$ st 1 disruption mutant when grown on autoclaved maize kernels and a number of synthetic media. The only discernible phenotype these authors observed was reduced $\mathrm{FB}_{1}$ production. Thus, we were surprised to observe reduced growth and symptom development when living kernels were inoculated with the mutant. In woundinoculated maize ears, the wild-type strain quickly colonized the endosperm tissues and rot symptoms were visible after a few days. The slower colonization of the kernel by strain $\Delta f s t 1$ indicates that the gene is required for normal pathogenicity. In contrast to colonization, $\mathrm{FB}_{1}$ production in the inoculated living kernels was similar to that observed in inoculated autoclaved kernels. Even by 14 days after inoculation, when the kernels infected by the mutant exhibited extensive disease symptoms, no $\mathrm{FB}_{1}$ was detectable. It was not possible to determine whether the lack of $\mathrm{FB}_{1}$ production by strain $\Delta f s t l$ in the maize kernel was directly responsible for the slower rate of colonization. However, previous studies have demonstrated that $\mathrm{FB}_{1}$ is not involved in the colonization of maize ears (Desjardins and Plattner 2000; Desjardins et al. 2002). These authors showed that $\mathrm{FB}_{1}$-nonproducing strains colonized maize kernels similar to wild type, $\mathrm{FB}_{1}$-producing strains.

A likely role of FST1 is in the metabolism of complex carbon resources in the host tissues that are encountered during colonization. To date, only a few genes involved in carbon metabolism have been shown to be pathogenicity genes (Rui and Hahn 2007; Wilson et al. 2007). Jobic and associates (2007) hypothesized that a hexose transporter gene hxtl in Sclerotinia sclerotiorum has a key role in pathogenesis. These authors used specific antibodies to show that invertase increased upon infection of sunflower cotyledons and that sucrose hydrolysis was coordinated with the expression of hxtl, which was highest $36 \mathrm{~h}$ after inoculation of sunflowers. The pathogen Uromyces fabae expresses a hexose transporter ( $h x t l)$ exclusively in the haustorium during infection but not in germ tubes, appressoria, infection hyphae, or haustorial mother cells (Voegele et al. 2001). Also, HXT1 was localized in the haustorial membrane. None of these transporters has high sequence similarity to FST1 $(<30 \%)$.

It has been postulated that some sugar transporters in filamentous fungi are involved in environmental sensing but their exact role in pathogenicity is not clearly understood. Experiments in yeast failed to demonstrate that FST1 has hexose transporter activity. The expression data indicate that a full-length transcript of $f s t l$ is produced in yeast strain YFST1. It is possible that the protein failed to function because of improper protein folding or improper insertion into the yeast membrane. However, the complementation by FST13 indicates that hexose transporters from $F$. verticillioides can function properly in yeast. Therefore, the negative results with YFST1 suggest that the protein may transport some other sugar type (di- or trisaccharides) or function as a sensor. Sugar sensors in yeast (Snf3p and Rgt2p) have long cytoplasmic C-termini that function in signal transduction. The C-termini of RCO3 of $N$. crassa and FST1 are considerably shorter than those in the yeast proteins. Lalonde and associates (1999) examined the topology of two putative monosaccharide transporters from Arabidopsis, AtSUGTRPR and F23E12.140, which have sensing functions similar to Rgt2p and Snf3p. Their analysis indicated that the large central loop of these proteins extended into the cytoplasm. A topological analysis of FST1 predicts that the protein contains 12 putative transmembrane domains with the $\mathrm{N}$ (74 residues) and $\mathrm{C}$ (64 residues) termini as well as a long central loop (70 residues) extending into the cytoplasm. Snf3p, Rgt2p, and RCO3 are also predicted to contain central loops within the cytoplasm (Table 3). Furthermore, the proteins with highest sequence similarity to FST1 in $F$. graminearum, $F$. oxysporum, $M$. grisea, and A. nidulans also have central loops that are predicted to be cytoplasmic. In contrast, the sugar transporters Hxt1p in Saccharomyces cerevisiae, FRT1 in Botrytis cinerea, and FST13 in F. verticillioides have long

Table 3. Central loop orientations of sugar transporters in fungi ${ }^{\mathrm{a}}$

\begin{tabular}{lll}
\hline Species & Protein & $\begin{array}{c}\text { Central loop } \\
\text { orientation }\end{array}$ \\
\hline Saccharomyces cerevisiae & Rgt2p & Cytoplasmic \\
S. cerevisiae & Snf3p & Cytoplasmic \\
Neurospora crassa & RCO3 & Cytoplasmic \\
S. cerevisiae & Hxt1p & External \\
Botrytis cinerea & FRT1 & External \\
Uromyces fabae & HXT1 & Cytoplasmic \\
Fusarium verticillioides & FST1 & Cytoplasmic \\
F. verticillioides & FST13 & External \\
\hline
\end{tabular}

a Analysis was conducted by TMHMM v. 2.0. 
central loops that extend externally. One exception is HXT1 in $U$. fabae, which has a central loop predicted to be cytoplasmic. Interestingly, a phylogenetic tree placed HXT1 into a clade with known sugar sensors (Voegele et al. 2001), suggesting that this protein, like $\mathrm{RCO} 3$, may have transporter activity as well as a sensor function. Whether or not the central loop orientation is a signature of a sugar sensor remains to be determined.

Shim and Woloshuk (1999) demonstrated that excess ammonium in the growth medium represses $\mathrm{FB}_{1}$ biosynthesis in $F$. verticillioides. Kim and Woloshuk (2008) showed that AREA, a global nitrogen metabolite regulator, is required for $\mathrm{FB}_{1}$ production during colonization of mature kernels. When grown on mature maize kernels, a constitutively expressed are $A$ strain produced $\mathrm{FB}_{1}$ even in the presence of high amounts of ammonium, which repressed $\mathrm{FB}_{1}$ biosynthesis in the wild type. Conserved GATA sites located in the promoter sequences of the fum genes suggest that they are regulated by AREA. Expression of $f s t l$ also is regulated by nitrogen source. With excess ammonium in the culture medium, expression of $f s t 1$ promoter:: $m$ Cherry-FP was repressed. Similar to the fum genes, the promoter region of $f$ st 1 has several putative AREA binding sites (-393, -737 , and $-862 \mathrm{bp}$ ), suggesting that $f_{s t} 1$ is regulated by AREA. Expression studies with the strain containing the $f$ st 1 promoter::mCherry-FP also confirmed observations made by Bluhm and associates (2008) that fstl is highly expressed in maize endosperm. These results indicate that $f$ st 1 is part of the same regulatory circuitry that responds to the nutritional status of the environment.

Expression of the fluorescently tagged FST1 suggests that the protein is localized in the plasma membrane. Furthermore, the abundance of fluorescence in vacuole-like vesicles suggests that either the fusion protein is unstable in the plasma membrane or FST1 is rapidly turned over. Both explanations may be valid based on the observation that the disappearance of fluorescence corresponded to sugar exhaustion and the extensive amount of fluorescence observed in vacuole-like vesicles, even while the fungus was growing on maize kernels. Regardless of the exact mechanism, these observations suggest that FST1 is internalized by endocytosis and, subsequently, degraded in vacuoles. Such a

Table 4. Primers used in this study

\begin{tabular}{ll}
\hline Primer name & \multicolumn{1}{c}{ Sequence 5'-3' } \\
\hline FST1-cDNAF & TTCACCATGGGCAAGAGTCG \\
FST1-cDNAR & CTCGAGTCAACCCATCCATCACCA \\
FST13-cDNAF & ATGAACGCTATTCGAAGGGCATCC \\
FST13-cDNAR & CCGACAGCAGAACTTCCAAACTCA \\
YHXT1-F & TTCCCCACCTTAAAATCTAT \\
YHXT1-R & CTCGAGGTGCCAGTCAGAAAATACAT \\
FST1-promF & CACGTTGGGCGCGGTATTTT \\
FST1-promR & CTCCTCGCCCTTGCTCACCATGGTGAAGAG \\
& ATGATAAT \\
Cherry-F & ATGGTGAGCAAGGGCGAGGAG \\
Cherry-R & GCAGCGAGTCAGTGAGCGAGGAA \\
Cherry-SF & GAGCAAGGGCGAGGAGGATAACAT \\
Cherry-SR & GGGGCCGTCGGAGGGGAAGT \\
FST1-promNestF & GCGGCCGCTTTCATTCAA \\
Cherry-NestR & GCGGCCGCGTCACTGGATT \\
FST1-p509 & CCTACCCACCGGACCGACACCTA \\
FST1-p247 & CGGCGAATTGTCGGTGCTA \\
FST1-p96 & GCCGCGACAAGTAATTCTTTCAAC \\
FST1-Cherry & CTCCTCGCCCTTGCTCACCATAGCATGAGA \\
& GACCTGGACCTGAT \\
FUM8-promF & TGCATGAACAATATAACGGAATCT \\
FUM8-promR & GATGGGCAATGCAAGGGCTG \\
FUM8-promNestF & CGTAAAGGTAGCAGCAAATGAAGG \\
FUM8-GFP & CAGCCCTTGCATTGCCCATCATGGTGAGCA \\
& AGGGCGAGGAG \\
GFP-R & GCTGCATGTGTCAGAGGTTTTCA \\
GFP-NestR & CGACCGGCGCTCAGTTGG \\
\hline
\end{tabular}

mechanism has been described for several yeast sugar transporters, including Hxt5p (van Suylekom et al. 2007). In addition, GFP fusions of Hxt2p, Hxt6p, and Hxt7p were degraded in vacuoles by a similar mechanism described in degradation of Hxt5p (Krampe and Boles 2002; Kruckeberg et al. 1999).

The results of this study indicate that $f_{s t} 1$ impacts the colonization of living maize kernels, affecting the development of disease symptoms. Furthermore, fumonisin production by the pathogen on a wide range of media requires a functional copy of $f s t 1$. Although fluorescently tagged FST1 was localized to the plasma membrane, there is no evidence that the protein functions as a hexose transporter as predicted by conceptual translation. Future studies will examine the significance of the central loop of the protein to determine whether FST1 functions as a sensor.

\section{MATERIALS AND METHODS}

\section{Fungal strains and culture conditions.}

F. verticillioides 7600 (M3125; Fungal Genetics Stock Center, Kansas City, KS, U.S.A.) and $\Delta f s t 1$ strain (Bluhm et al. 2008) were stored at $-80^{\circ} \mathrm{C}$ in $20 \%$ glycerol and grown on potato dextrose agar (PDA; B\&D, Sparks, MD, U.S.A.) for inoculum. Cultures of autoclaved maize kernels (inbred B73) were prepared as previously described by Bluhm and Woloshuk (2005). Yeast strain EBY.VW4000 (CEN.PK2-1C $4 h x t 1-17$ $\Delta$ gal2 $\Delta$ stl1 $\Delta$ agtl $\Delta m p h 2 \Delta m p h 3)$ (Wieczorke et al. 1999) was maintained on synthetic medium (SC) plus $2 \%$ maltose. The $\mathrm{SC}$ medium consists of $1.7 \mathrm{~g}$ of yeast nitrogen base without amino acids (Difco Laboratories, Detroit), $5 \mathrm{~g}$ of ammonium sulfate, $20 \mathrm{mg}$ of L-trytophan, $20 \mathrm{mg}$ of histidine, $60 \mathrm{mg}$ of Lleucine, $20 \mathrm{mg}$ of uracil, and $20 \mathrm{~g}$ of agar per liter.

\section{Nucleic acid manipulation and fungal transformation.}

Bacterial plasmids were isolated with a Qiagen Miniprep DNA Purification System (Qiagen, Valencia, CA, U.S.A.). Fungal genomic DNA was isolated as described previously (Shim and Woloshuk 2001). PCR primers were obtained from Integrated DNA Technologies (Coralville, IA, U.S.A.) and are listed in Table 4. For the fungal transformations, the methods used to prepare protoplasts and to regenerate transformants were performed as described previously (Shim and Woloshuk 2001).

\section{Expression of $f$ st 1 in yeast.}

Gene fstl (GenBank accession no. EU152990) cDNA was amplified by primer pair FST1-cDNAF and FST1-cDNAR (Table 4) from total RNA and cloned into pGEM-T Easy vector (Promega Corp., Madison, WI, U.S.A.). fst 1 cDNA was excised from the pGEM-T Easy with SpeI/XhoI and cloned into p427TEF (Dualsystems Biotech Inc., San Carlos, CA, U.S.A.), which is a yeast expression vector with the translation elongation factor $(T E F 1)$ gene promoter, cytochrome c $(C Y C 1)$ gene terminator, and the geneticin-resistance marker gene. The resulting construct, pTEF::FST1, was transformed into yeast mutant EBY.VW4000 by an alkali-cation yeast transformation kit (Qbiogene, Carlsbad, CA, U.S.A.). Gene $f s t 13$ (GenBank accession no. GU947704) cDNA and yeast HXT1 (GenBank accession no. M82936) were amplified by primer pairs FST13cDNAF/FST13-cDNAR and YHXT1-F/YHXT1-R (Table 4), respectively, and also cloned into p427TEF by the same method used for $f s t 1$ cDNA. The correct sequences for all cDNA expression constructs were verified by DNA sequencing. Yeast transformants were selected on SC medium containing $20 \mathrm{~g}$ of maltose and $200 \mathrm{mg}$ of geneticin per liter. The growth of selected yeast transformants was tested on YEP media (3 $\mathrm{g}$ of yeast extract and $10 \mathrm{~g}$ of peptone per liter) containing $20 \mathrm{~g}$ of glucose, fructose, mannose, or maltose per liter, and the cultures were 
photographed after 3 days of incubation at $30^{\circ} \mathrm{C}$. To confirm expression of $f s t 1$ in yeast, cells were harvested from $10 \mathrm{ml}$ of culture (optical density at $600 \mathrm{~nm}$ of approximately 1.0) growing on a YEP $+2 \%$ maltose medium. Total RNA was extracted with TRIzol reagent (Invitrogen, Carlsbad, CA, U.S.A.) by the manufacturer's protocol, and cDNA was generated as described by Flaherty and associates (2003). From the cDNA, the full length of $f$ st 1 was amplified by primer pair FST1-cDNAF and FST1cDNAR. PCR cycling conditions were $2 \mathrm{~min}$ at $94^{\circ} \mathrm{C}$; followed by 34 cycles of $30 \mathrm{~s}$ at $94^{\circ} \mathrm{C}, 30 \mathrm{~s}$ at $58^{\circ} \mathrm{C}$, and $90 \mathrm{~s}$ at $72^{\circ} \mathrm{C}$; and $10 \mathrm{~min}$ at $72^{\circ} \mathrm{C}$ for a final extension.

\section{fst1 promoter activity.}

The $f s t 1$ promoter $(0.9 \mathrm{~kb})$ was PCR amplified from genomic DNA of $F$. verticillioides 7600 with primers FST1-promF and FST1-promR (Table 4) and fused to DNA fragment mCherry$F P(0.7 \mathrm{~kb})$, which is the coding region (including $3^{\prime}$ flanking region) of a red fluorescent protein obtained from pSAT4AmCherry-N1 with primers Cherry-F and Cherry-R (Table 4). After fusion of the two fragments, the resulting PCR product (1.6 kb) was amplified with primers FST1-promNestF and CherryNestR (Table 4). This PCR product was cloned into the pGEM$\mathrm{T}$ easy vector to create $\mathrm{pFPC} 4$ and co-transformed into wildtype strains with pKS-HYG carrying a hygromycin-resistance gene. Twenty transformants were screened by PCR with two primer pairs, Cherry-SF/Cherry-SR (internal region of $m$ CherryFP) (Table 4) and FST1-promNestF/Cherry-NestR. The PCR results showed that there were three independent transformants containing $f_{s t} l$ promoter fused to $m C h e r r y-F P$, and the expression of mCherry-FP was confirmed; one selected strain was designated as FPC-896. The PCR conditions were 2 min at $94^{\circ} \mathrm{C}$; followed by 30 cycles of $30 \mathrm{~s}$ at $94^{\circ} \mathrm{C}, 30 \mathrm{~s}$ at $58^{\circ} \mathrm{C}$, and $90 \mathrm{~s}$ at $72^{\circ} \mathrm{C}$; and $10 \mathrm{~min}$ at $72^{\circ} \mathrm{C}$ for a final extension. To determine the crucial region for $f s t 1$ promoter activity, three expression constructs were generated, each with a different size of $f s t l$ promoter fused to $m$ Cherry-FP. The constructs were made by PCR with pFPC4 as the template and the primer pairs FST1-p509/ Cherry-NestR, FST1-p247/Cherry-NestR, and FST1-p96/ Cherry-NestR (Table 4). The amplified PCR products were transformed into the wild-type strain with $\mathrm{pKS}-\mathrm{HYG}$. The same strategy described for strain FPC-896 was used to screen for transformants. We obtained at least two independent strains of each construct and observed the mCherry-FP expression.

To test the effects of nitrogen source on $f s t l$ expression, strain FPC-896 was grown in YEP medium at $28^{\circ} \mathrm{C}$. After 4 days, the cultures were centrifuged, and the mycelial pellets were transferred to $20 \mathrm{ml}$ of DLG ( $3 \mathrm{~g}$ of potassium phosphate, $0.3 \mathrm{~g}$ of magnesium sulfate, $5 \mathrm{~g}$ of sodium chloride, $1 \mathrm{~g}$ of bovine serum albumin, and $20 \mathrm{~g}$ of glucose per liter). To maintain high nitrogen conditions, $400 \mu \mathrm{l}$ of $1 \mathrm{M}$ ammonium phosphate was added every $24 \mathrm{~h}$ to the DLG cultures. Two strains were used as controls: a wild-type strain containing NIAD-GUS (Kim and Woloshuk 2008) and a wild-type strain expressing GFP driven by the fum 8 (GenBank accession no. AF155773) promoter. For the latter strain, the fum 8 promoter region was amplified from genomic DNA of $F$. verticillioides 7600 with primers FUM8promF and FUM8-promR (Table 4) and fused to a GFP fragment that was amplified from pEGFP (Clontech Laboratories, Inc., Mountain View, CA, U.S.A.) with primers FUM8-GFP and GFP-R (Table 4). The final construct $(1.5 \mathrm{~kb})$ was amplified from the fused fragments with primers FUM8-promNestF and GFP-NestR (Table 4) and cloned into pGEM-T Easy vector. This clone was co-transformed into wild-type protoplasts with pKS-HYG. To monitor the expression of reporter genes ( $m$ Cherry-FP and GFP), an epifluorescence microscope Eclipse E 800 (Nikon, Melville, NY, U.S.A.) was used. GUS activity was assayed as described previously (Kim and Woloshuk 2008).

\section{Localization of FST1.}

The $m$ Cherry-FP gene was fused in-frame to the $3^{\prime}$ end of $f s t 1$. The $f s t 1$ and $m C h e r r y-F P$ genes were amplified by primer pairs FST1-promF/FST1-Cherry and Cherry-F/Cherry-R (Table 4 ), respectively. These two PCR products were fused with a single-joint PCR technique modified from $\mathrm{Yu}$ and associates (2004) and cloned into the pGEM-T easy vector. For the final PCR product, primers FST1-promNestF and Cherry-NestR (Table 4) were used, and PCR conditions were $2 \mathrm{~min}$ at $94^{\circ} \mathrm{C}$; followed by 30 cycles of $30 \mathrm{~s}$ at $94^{\circ} \mathrm{C}, 30 \mathrm{~s}$ at $58^{\circ} \mathrm{C}$, and $3 \mathrm{~min}$ at $72^{\circ} \mathrm{C}$; and $10 \mathrm{~min}$ at $72^{\circ} \mathrm{C}$ for a final extension. Also, a benA terminator from GAP4 (Woloshuk and Payne 1994) was inserted into the C-terminus of $m$ Cherry-FP at the SacI restriction enzyme site. The resulting vector was designated as pFCT11 (fst $1:: m C h e r r y-F P::$ benA terminator) and used directly along with pKS-GEN, which carries the geneticin-resistance gene as a selectable marker, to co-transform protoplasts of strain $\Delta f s t 1$. A transformant designated as $\Delta f s t 1$-FCT11 was identified and analyzed. An LSM710 (Laser Scanning Microscopes; Carl Zeiss, Jena, Germany) with Plan-Apochromat objective lenses $(\times 20, \times 63 / 1.40$ oil, and $\times 100 / 1.40$ oil $)$ was used to observe the fungal mycelia. Strain $\Delta f s t 1$-FCT11 was grown on YEP medium for 2 days, at which time the fungal mycelia were transferred into DLG (glucose medium) or defined liquid medium with $2 \%$ amylopectin instead of glucose as the carbon source (amylopectin medium). Observations with the confocal microscope were made at $6,24,48,72$, and $96 \mathrm{~h}$ after transferring mycelia. When grown on mature kernels prepared as described previously by Bluhm and Woloshuk (2005), the observations were performed at 4 and 7 days after inoculation.

\section{Pathogenicity assay.}

Seed of maize inbred line B73 were sown in greenhouse beds and fields at the Purdue University Agronomy Center for Research and Education (West Lafayette, IN, U.S.A.). Plants were self pollinated and ears were removed at the dough stage (R4) of development (approximately 28 days after pollination). For the pathogenicity assay, the ear husk was pulled back and the silks were removed prior to inoculation. For each strain, the ears were wound inoculated with a pin-bar (18 pins; $0.5 \mathrm{~cm}$ in depth) that was dipped into a suspension of $10^{6}$ conidia $/ \mathrm{ml}$. After inoculation of 5 to 10 rows per ear, the husks were placed back into position and secured with a rubber band. The ears were incubated in clear containers within the greenhouse $\left(25^{\circ} \mathrm{C}\right.$ with high humidity) for 7 or 14 days. The pathogenicity assays were performed with three independent biological repetitions, and the experiment was repeated three times with similar results. Ten wound-inoculated kernels were collected randomly from each ear, and ground kernels with liquid $\mathrm{N}_{2}$ were used for $\mathrm{FB}_{1}$ and ergosterol analysis.

\section{$\mathrm{FB}_{1}$ and ergosterol analysis.}

$\mathrm{FB}_{1}$ from colonized maize kernels was analyzed by methods described by Kim and Woloshuk (2008). To analyze $\mathrm{FB}_{1}$ from liquid cultures, $4 \mathrm{ml}$ of culture was centrifuged for $10 \mathrm{~min}$, and the supernatant was passed through a C-18 solid-phase extraction column (Agilent Technologies, Palo Alto, CA, U.S.A.) as described by Shim and Woloshuk (1999). FB 1 was eluted with $2 \mathrm{ml}$ of $70 \%$ acetonitrile and analyzed by the same method used for $\mathrm{FB}_{1}$ from colonized maize kernels. Fungal growth was quantified by analysis of ergosterol content from colonized maize kernels, as described by Kim and Woloshuk (2008).

\section{Sugar analysis.}

Total sugar content was measured by a modified anthrone method (Laurentin and Edwards 2003). Briefly, the wild-type strain was grown on YEP medium for 3 days, and the mycelial 
mass was transferred to $20 \mathrm{ml}$ of glucose medium or amylopectin medium. From two cultures, $100 \mu \mathrm{l}$ of culture medium was obtained at $0,24,48,72$, and $96 \mathrm{~h}$. These samples were diluted 200-fold with water for the analysis. Samples $(300 \mu \mathrm{l})$ were mixed with $600 \mu \mathrm{l}$ of anthrone reagent (anthrone at 2 $\mathrm{mg} / \mathrm{ml}$ of sulfuric acid) in test tubes. After $5 \mathrm{~min}$ in a boiling water bath, absorbance at $630 \mathrm{~nm}$ was measured with a spectrophotometer (Beckman Instruments Inc., Fullerton, CA, U.S.A.). A standard curve was prepared with glucose.

\section{ACKNOWLEDGMENTS}

We thank B. Bluhm, L. Dunkle, and G. Payne for their helpful discussions and reviews of this manuscript; E. Boles (Goethe University, Frankfurt) for providing the yeast strain EBY.VW4000; and S. Loesch-Fries (Purdue University, West Lafayette) for the plasmid pSAT4A-mCherryN1. Support for this research was provided by the United States Department of Agriculture National Institute of Food and Agriculture (NIFA) award number 10-65108-20567.

\section{LITERATURE CITED}

Bluhm, B. H., and Woloshuk, C. P. 2005. Amylopectin induces fumonisin B1 production by Fusarium verticillioides during colonization of maize kernels. Mol. Plant-Microbe Interact. 18:1333-1339.

Bluhm, B. H., Kim, H., Butchko, R. A. E., and Woloshuk, C. P. 2008. Involvement of ZFR1 of Fusarium verticillioides in kernel colonization and the regulation of FST1, a putative sugar transporter gene required for fumonisin biosynthesis on maize kernels. Mol. Plant Pathol. 9:203211.

Brown, D. W., Cheung, F., Proctor, R. H., Butchko, R. A., Zheng, L., Lee, Y., Utterback, T., Smith, S., Feldblyum, T., Glenn, A. E., Plattner, R. D., Kendra, D. F., Town, C. D., and Whitelaw, C. A. 2005. Comparative analysis of 87,000 expressed sequence tags from the fumonisin-producing fungus Fusarium verticillioides. Fungal Genet. Biol. 42:848-861.

Desai, K., Sullards, M. C., Allegood, J., Wang, E., Schmelz, E. M., Hartl, M., Humpf, H. U., Liotta, D. C., Peng, Q., and Merrill, A. H., Jr. 2002. Fumonisins and fumonisin analogs as inhibitors of ceramide synthase and inducers of apoptosis. Biochim. Biophys. Acta 1585:188192

Desjardins, A. E., and Plattner, R. D. 2000. Fumonisin $\mathrm{B}_{1}$-nonproducing strains of Fusarium verticillioides cause maize (Zea mays) ear infection and ear rot. J. Agric. Food Chem. 48:5773-5780.

Desjardins, A. E., Munkvold, G. P., Plattner, R. D., and Proctor, R. H. 2002. FUM1-a gene required for fumonisin biosynthesis but not for maize ear rot and ear infection by Gibberella moniliformis in field tests. Mol. Plant-Microbe Interact. 15:1157-1164.

Flaherty, J. E., Pirttila, A. M., Bluhm, B. H., and Woloshuk, C. P. 2003. PAC1, a pH-regulatory gene from Fusarium verticillioides. Appl. Environ. Microbiol. 69:5222-5227.

Jobic, C., Boisson, A. M., Gout, E., Rascle, C., Fevre, M., Cotton, P., and Bligny, R. 2007. Metabolic processes and carbon nutrient exchanges between host and pathogen sustain the disease development during sunflower infection by Sclerotinia sclerotiorum. Planta 226:251-265.

Kim, H., and Woloshuk, C. P. 2008. Role of AREA, a regulator of nitrogen metabolism, during colonization of maize kernels and fumonisin biosynthesis in Fusarium verticillioides. Fungal Genet. Biol. 45:947-953.

Krampe, S., and Boles, E. 2002. Starvation-induced degradation of yeast hexose transporter Hxt7p is dependent on endocytosis, autophagy and the terminal sequences of the permease. FEBS (Fed. Eur. Biochem. Soc.) Lett. 513:193-196.
Kruckeberg, A. L., Ye, L., Berden, J. A., and van Dam, K. 1999. Functional expression, quantification and cellular localization of the Hxt2 hexose transporter of Saccharomyces cerevisiae tagged with the green fluorescent protein. Biochem. J. 339:299-307.

Lalonde, S., Boles, E., Hellmann, H., Barker, L., Patrick, J. W., Frommer, W. B., and Ward, J. M. 1999. The dual function of sugar carriers. Transport and sugar sensing. Plant Cell 11:707-726.

Laurentin, A., and Edwards, C. A. 2003. A microtiter modification of the anthrone-sulfuric acid colorimetric assay for glucose-based carbohydrates. Anal. Biochem. 315:143-145.

Madi, L., McBride, S. A., Bailey, L. A., and Ebbole, D. J. 1997. rco-3, a gene involved in glucose transport and conidiation in Neurospora crassa. Genetics 146:499-508.

Ozcan, S., Dover, J., Rosenwald, A. G., Wolfl, S., and Johnston, M. 1996. Two glucose transporters in Saccharomyces cerevisiae are glucose sensors that generate a signal for induction of gene expression. Proc. Natl. Acad. Sci. U.S.A. 93:12428-12432.

Ozcan, S., Dover, J., and Johnston, M. 1998. Glucose sensing and signaling by two glucose receptors in the yeast Saccharomyces cerevisiae. EMBO (Eur. Mol. Biol. Organ.) J. 17:2566-2573.

Rheeder, J. P., Marasas, W. F., and Vismer, H. F. 2002. Production of fumonisin analogs by Fusarium species. Appl. Environ. Microbiol. 68:2101-2105

Rui, O., and Hahn, M. 2007. The Botrytis cinerea hexokinase, Hxk1, but not the glucokinase, Glk1, is required for normal growth and sugar metabolism, and for pathogenicity on fruits. Microbiology 153:2791-2802.

Santangelo, G. M. 2006. Glucose signaling in Saccharomyces cerevisiae. Microbiol. Mol. Biol. Rev. 70:253-282.

Shim, W. B., and Woloshuk, C. P. 1999. Nitrogen repression of fumonisin B1 biosynthesis in Gibberella fujikuroi. FEMS (Fed. Eur. Microbiol. Soc.) Microbiol. Lett. 177:109-116.

Shim, W. B., and Woloshuk, C. P. 2001. Regulation of fumonisin B1 biosynthesis and conidiation in Fusarium verticillioides by a cyclin-like (C-type) gene, FCC1. Appl. Environ. Microbiol. 67:1607-1612.

Shim, W. B., Flaherty, J. E., and Woloshuk, C. P. 2003. Comparison of fumonisin B1 biosynthesis in maize germ and degermed kernels by Fusarium verticillioides. J. Food Prot. 66:2116-2122.

van Suylekom, D., van Donselaar, E., Blanchetot, C., Do Ngoc, L. N., Humbel, B. M., and Boonstra, J. 2007. Degradation of the hexose transporter Hxt5p in Saccharomyces cerevisiae. Biol. Cell 99:13-23.

Voegele, R. T., Struck, C., Hahn, M., and Mendgen, K. 2001. The role of haustoria in sugar supply during infection of broad bean by the rust fungus Uromyces fabae. Proc. Natl. Acad. Sci. U.S.A. 98:8133-8138.

Wieczorke, R., Krampe, S., Weierstall, T., Freidel, K., Hollenberg, C. P., and Boles, E. 1999. Concurrent knock-out of at least 20 transporter genes is required to block uptake of hexoses in Saccharomyces cerevisiae. FEBS (Fed. Eur. Biochem. Soc.) Lett. 464:123-128.

Wilson, R. A., Jenkinson, J. M., Gibson, R. P., Littlechild, J. A., Wang, Z. Y., and Talbot, N. J. 2007. Tps1 regulates the pentose phosphate pathway, nitrogen metabolism and fungal virulence. EMBO (Eur. Mol. Biol. Organ.) J. 26:3673-3685.

Woloshuk, C. P., and Payne, G. A. 1994. The alcohol dehydrogenase gene adhl is induced in Aspergillus flavus grown on medium conducive to aflatoxin biosynthesis. Appl. Environ. Microbiol. 60:670-676.

Yu, J. H., Hamari, Z., Han, K. H., Seo, J. A., Reyes-Dominguez, Y., and Scazzocchio, C. 2004. Double-joint PCR: a PCR-based molecular tool for gene manipulations in filamentous fungi. Fungal Genet. Biol. 41:973-981.

\section{AUTHOR-RECOMMENDED INTERNET RESOURCES}

The Broad Institute website: www.broadinstitute.org

Center for Biological Sequence Analysis TMHMM prediction server: www.cbs.dtu.dk/services/TMHMM 